shows no change. Finally, a synthetic miR-21 mimic and inhibitor were used to regulate the miR-21 expression in the RVMC, and a synthetic siRNA of PDCD4 is used at the same time. Transfected with miR-21 mimic could decrease the expression of PDCD4, caspase-3 activity and protein expression, TUNEL assay also shows a decreased number of apoptosis cells, while transfected siRNA PDCD4 together with miR-21 mimic, the anti-apoptosis function of miR-21 disappeared.

Conclusions AngII induced a different result in the RVMC compared with the LVMC. AngII induced hypertrophy in the LVMC while accompany apoptosis in the RVMC. MiR-21 expression changes differently in the LVMC and RVMC, miR-21 protect the RVMC from apoptosis under AngII stimulus via targeting PDCD4 but not FasL.

\section{[gw22-e0628] MIR-21 PROTECTS THE RIGHT VENTRICLE MYOCARDIAL CELLS FROM APOPTOSIS UNDER THE ANG II STIMULUS VIA TARGETING PDCD4}

Fang Liu, Jian Wu, Li Yin, Maomao Zhang Department Of Cardiology, The Second Affiliated Hospital Of Harbin Medical University, Harbin, China

10.1136/heartjnl-2011-300867.220

Background Evidence showed that the left ventricle and right ventricle are composed of cells with unique original, functional, structural, metabolic and electrophysiological characteristics, and the two ventricles have different hemodynamics and pathophysiological characteristics. Therefore, right ventricular failure cannot be understood simply by extrapolating data and experience from left ventricular failure. But, till now the mechanism of the right ventricle failure is still unclear, and there are still no studies about the right ventricle cells.

Methods and Results First, we contrast the changes between the right and left myocardial cells after different concentration of AngII (100 nM, $1000 \mathrm{nM}, 1000 \mathrm{nM}$ ) stimulation. ANF mRNA was detected to evaluate the myocardial cells hypertrophy. Both the right and left cardiac myoctyes show an increasing expression of ANF mRNA, but a converse tendency. As the concentration of AngII increasing, ANF mRNA expression in the left $\mathrm{s}$ is increasing while decreasing in the RVMC. The sign of apoptosis was evaluated by electron microscope, TUNEL assay, caspase- 3 activity and protein expression. After AngIIstimulation, the right myocardial cells showed early stage of apoptosis in all of the AngII groups, while the left myocardial cells showed to be normal. Secondly, in order to find the hidden mechanism, anti-apoptosis microRNA, miR-21 was detected through qRT-PCR. In contrast to the LVMC, the baseline expression of miR-21 in the RVMC is almost three times higher, and after AngII stimulation, it decreased significantly, while there is no change in the LVMC. FasL and PDCD4 are currently identified as a target of miR-21. Western blot was used to detect both of the protein expression after AngII stimulation. PDCD4 is increased accordingly while FasL 